\title{
Survival benefits associated with surgery for advanced non-small cell lung cancer
}

Elizabeth A. David, MD, MAS,,${ }^{\mathrm{a} b}$ Stina W. Andersen, $\mathrm{PhD},{ }^{\mathrm{c}}$ Laurel A. Beckett, $\mathrm{PhD},{ }^{\mathrm{d}}$

Joy Melnikow, MD MPH, ${ }^{\mathrm{c}}$ James M. Clark, MD, ${ }^{\mathrm{a}}$ Lisa M. Brown, MD, MAS, ${ }^{a}$ David T. Cooke, MD, Karen Kelly, MD, ${ }^{e}$ and Robert J. Canter, MD, MAS ${ }^{\mathrm{e}}$

\section{ABSTRACT}

Objective: Overall survival (OS) for advanced stage (IIIA-IV) non-small cell lung cancer (NSCLC) is highly variable, and retrospective data show a survival advantage for patients receiving therapeutic intent pulmonary resection. We hypothesized that this variability in OS can be modeled separately by stage to allow a personalized estimate of OS.

Methods: In a cohort of patients with advanced-stage NSCLC from the National Cancer Database, we assessed the accuracy of Surgical Selection Score (SSS) to predict OS using Cox proportional hazards models and determined by stage the effect of surgery on survival among people with similarly high levels of SSS.

Results: In total, 300,572 patients were identified; 18,701 (6\%) had surgery. The SSS was a strong predictor of OS (C-index, $0.89 ; 95 \%$ confidence interval [CI], $0.89-0.90)$. We observed significantly greater OS $(P<.001)$ among patients who had surgery. The hazard of death was at least 2 times greater for patients in the upper quartile of SSS who did not receive surgery compared with surgical patients even when adjusting for the SSS (stage IIIA: hazard ratio [HR], 2.1; 95\% CI, 2.02.2, stage IIIB: HR, 2.3; 95\% CI, 2.2-2.5, stage IV: HR, 2.3; 95\% CI, 2.2-2.4).

Conclusions: The SSS is highly predictive of individual OS and can be used as a risk assessment tool. These findings are important for a more robust evaluation of the likely benefits of surgical resection for these patients. After further prospective validation, the SSS can be used during treatment decision-making for patients with advanced-stage NSCLC. (J Thorac Cardiovasc Surg 2019;157:1620-8)

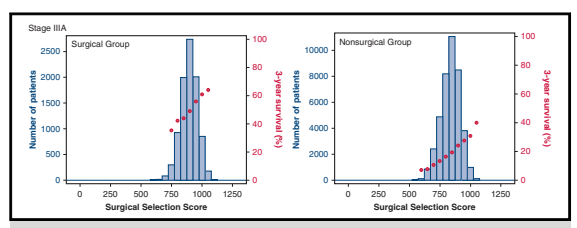

Patients who undergo lung resection live longer than those treated nonsurgically.

\section{Central Message}

When patients with advanced-stage NSCLC are treated with surgery, they live longer. This analysis demonstrates that this finding is not purely selection bias.

\section{Perspective}

We used a prediction model to assess the therapeutic impact of lung resection on overall survival. We demonstrate that across all stages, the hazard of death is twice as high in patients not treated surgically compared with surgically treated patients. Our model can be used to identify patients who have the greatest probability of benefiting from lung resection and to identify patients for whom lung resection has minimal opportunity to improve survival.

See Commentaries on pages 1629 and 1631.
Non-small cell lung cancer (NSCLC) is the leading cause of cancer-related mortality in the United States. ${ }^{1}$ Overall 5 -year survival is approximately $18 \%$, but for patients with advanced-stage (stages IIIA, IIIB, or IV) NSCLC, it is particularly poor. Importantly, however, survival rates for these subgroups are quite variable.

From the ${ }^{\text {a Section }}$ of General Thoracic Surgery and Outcomes Research Group, Department of Surgery and ${ }^{\mathrm{e}} \mathrm{UC}$ Davis Comprehensive Cancer Center, UC Davis Health, Sacramento, Calif; ${ }^{\mathrm{b}}$ Heart Lung Vascular Center, David Grant Medical Center, Travis AFB; ${ }^{\circ}$ Center for Healthcare Policy and Research, UC Davis, Sacramento; and ${ }^{\mathrm{d}}$ Department of Public Health Sciences, UC Davis School of Medicine, Davis. The project described was supported by the National Center for Advancing Translational Sciences, National Institutes of Health, through grant number UL1 TR000002. The content is solely the responsibility of the authors and does not necessarily represent the official views of the National Institutes of Health. This work was directly supported by the Department of Surgery Outcomes Research Group (E.A.D.), an American Cancer Society Institutional Research Grant
Depending on factors such as stage of disease and treatment modality, 5 -year survival ranges from $4 \%$ to $28 \%{ }^{2}$ The heterogeneity of the clinical, pathologic, and facility characteristics of these patients likely accounts, in part, for the variable survival outcomes among these patients, but these same variable characteristics also have been

(E.A.D.: ACS IRG-95-125-13), and the Christine and Helen Langraf Memorial Fund (E.A.D.)

Read at the 98th Annual Meeting of The American Association for Thoracic Surgery, San Diego, California, April 28-May 1, 2018.

Received for publication Jan 15, 2018; revisions received Sept 26, 2018; accepted for publication Oct 2, 2018; available ahead of print Dec 11, 2018

Address for reprints: Elizabeth A. David, MD, MAS, 2221 Stockton Blvd, Rm 2121, Sacramento, CA 95817 (E-mail: elizabethadavid@gmail.com). 0022-5223/\$0.00

Published by Elsevier Inc. on behalf of The American Association for Thoracic Surgery

https://doi.org/10.1016/j.jtcvs.2018.10.140 


\section{Abbreviations and Acronyms \\ AJCC = American Joint Committee on Cancer \\ $\mathrm{CI}=$ confidence interval \\ $\mathrm{HR}=$ hazard ratio \\ NCDB = National Cancer Database \\ NSCLC $=$ non-small cell lung cancer \\ OS = overall survival \\ SSS $=$ Surgical Selection Score}

tumor size, American Joint Committee on Cancer (AJCC) clinical T status, AJCC clinical N status, AJCC clinical M status, Charlson comorbidity index, age, race, facility type, insurance, and income, with AJCC clinical M status, AJCC clinical N status, and age having the strongest influence on selection for surgery. In the current analysis, our objectives were to define the stage-specific ability of the SSS to predict overall survival (OS) for patients with advancedstage NSCLC to assess the impact of surgery in patients at same stage and comparable SSS and to provide a risk assessment tool.

implicated as the foundation of widely disparate treatment approaches. ${ }^{2,3}$ Moreover, it is these patients with advanced stage who make up the vast majority of patients with NSCLC, representing roughly $79 \%$ of newly diagnosed patients, so reduction in treatment variation and/or risk stratification is anticipated to translate to significant therapeutic gains.

Current National Comprehensive Cancer Network guidelines only recommend therapeutic intent surgical treatment for patients with stage IIIA disease who have single-station nodal disease or stage IV patients with oligometastatic disease to the brain or adrenal glands. ${ }^{4}$ Despite this limited recommendation for surgical treatment, several series have demonstrated a clinically and statistically significant survival advantage for patients treated surgically compared with patients treated nonsurgically. ${ }^{3,5,6}$ However, uniformly, the analyses demonstrating a survival advantage with surgery are retrospective in nature, and the survival advantages observed are typically attributed to selection bias in the surgical cohorts. ${ }^{3,5,6}$ An accepted approach to control for selection bias in retrospective analyses is to use statistical methodology such as propensity matching to control for confounding variables. However, this technique is limited by the inability to assess unmeasured confounders such as severity of comorbidities and disease burden. ${ }^{7}$ In addition, these techniques are typically limited to retrospective analyses of outcomes and do not directly assist with prospective treatment decision-making., ${ }^{5,6}$

In an effort to improve data-driven treatment decision making for these patients, we recently developed a Surgical Selection Score (SSS) to explore factors inherent in the selection for surgical treatment among advanced-stage NSCLC. ${ }^{8}$ One distinct advantage of the SSS over propensity matching is that it can be used in a prospective manner when treatment decisions are being made. The SSS was created in a population cohort from patients with advanced-stage NSCLC in the National Cancer Database (NCDB) using a logistic regression model that predicts likelihood of selection for surgery based on clinical factors available at the time of treatment decisions. The factors included in the SSS are all clinical, including histology,

\section{METHODS}

Using the NCDB Participant User File from 1998 to 2012, we identified cases of biopsy-proven NSCLC. The NCDB is a joint program of the Commission on Cancer and the American Cancer Society. ${ }^{9}$ Data from the NCDB represent 1500 Commission on Cancer-accredited facilities including more than $70 \%$ of all newly diagnosed cancer cases in the United States. ${ }^{9}$

Patients with clinical stage IIIA, IIIB, and IV NSCLC per the 7th edition of the International Association for the Study of Lung Cancer with histologic data available were included (Figure 1). Patients with an additional cancer diagnosis, missing clinical TNM stage group, clinical stage I or II, or missing Charlson index or other demographic data were excluded. Standard patient, tumor, and treatment data were extracted and categorized as appropriate. Patients were categorized into surgical and nonsurgical groups. Nonsurgical treatments included chemotherapy only, chemotherapy and radiation, radiation only, and no treatment. Surgical treatments included surgery only; chemotherapy and surgery; chemotherapy, radiation, and surgery; and surgery and radiation. Surgical operations included wedge resection, sublobar resection, lobectomy, bilobectomy, and pneumonectomy in an attempt to focus our analysis on therapeutic intent procedures aimed at resection of the primary tumor.

Patient comorbidities were assessed using the Charlson comorbidity index, described by Deyo and colleagues. ${ }^{10}$ Additional categorical variables examined included clinical tumor group, clinical $\mathrm{T}$ group, clinical $\mathrm{N}$ group, clinical M group, histology, age, sex, race, income, education, insurance status (Medicaid, Medicare, other government, private health insurance, no insurance), and treatment facility. Age was categorized by percentile (10th, 25th, 50th, 75th, 90th). Income categories were defined as follows:

\section{Statistical Analyses}

As we have previously described, logistic regression was used to create the SSS with the outcome variable representing inclusion of surgery in the treatment regimen. ${ }^{8}$ The SSS was created by a linear rescaling of the logarithm of the odds ratio of inclusion of surgery in the treatment regimen to generate a single numeric value between 0 and 1200 for each patient depending on the value of the clinical factors for each patient. In the current analysis, the ability of the SSS to predict OS was assessed using the Cox proportional hazards model with SSS as the only explanatory variable, for the entire cohort and separately by stage. Discrimination ability was measured by calculating an overall C-index (concordance index) for the Cox model. ${ }^{11,12}$ The C-index was introduced by Harrell as a natural extension of the area under the receiver operator curve from logistic regression. ${ }^{13}$ The $\mathrm{C}$-index is defined as the proportion of all usable patient pairs in which the predictions and outcomes are concordant. The overall C-index and confidence interval (CI) hereof for survival analysis where censoring is an issue was calculated as proposed by Pencina and D'Agostino. ${ }^{11,12}$

OS were illustrated and median survival times calculated using a Kaplan-Meier life table approach and compared by log-rank test. For low $\leq \$ 38,000$; middle $>\$ 38,000-47,999$, and high $\geq \$ 48,000$. 


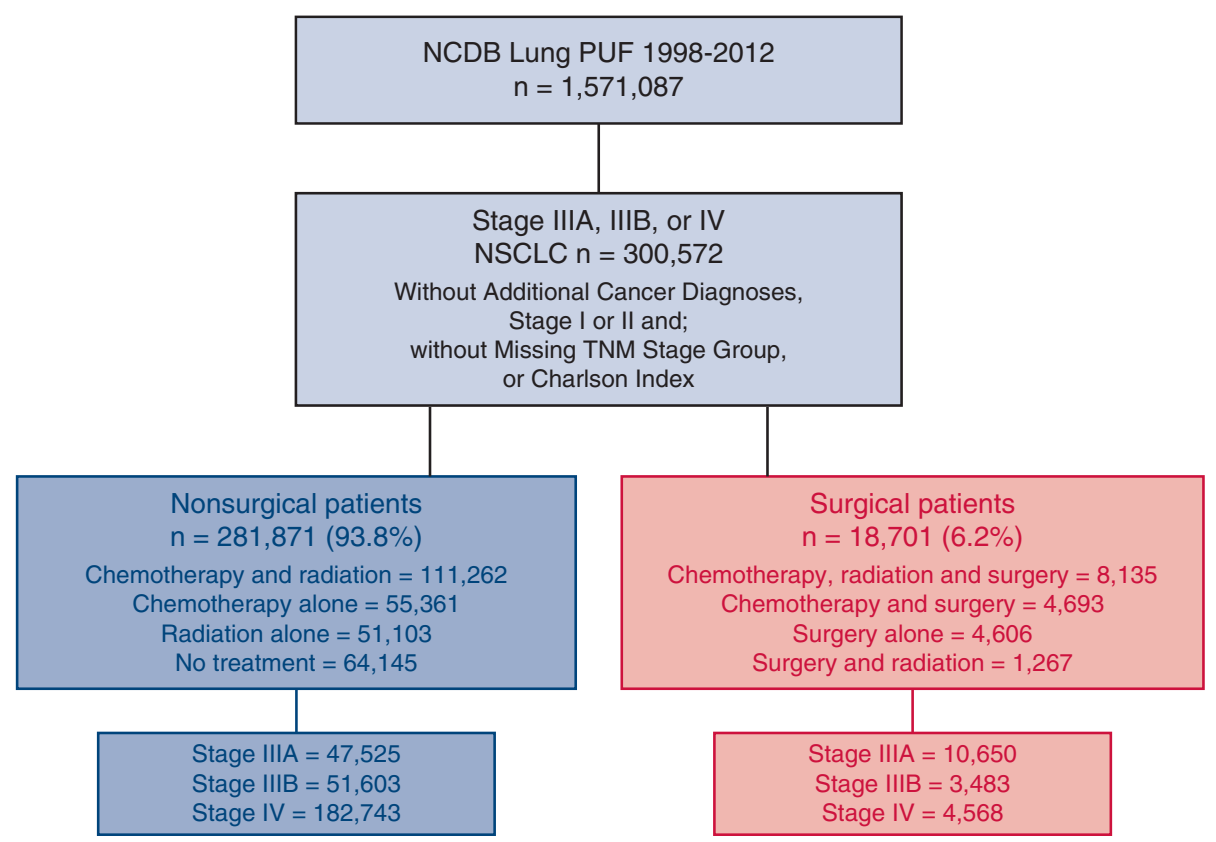

FIGURE 1. Cohort of patients with stage IIIA, IIIB, and IV NSCLC from the NCDB. NSCLC, Non-small cell lung cancer; NCDB, National Cancer Database; $P U F$, participant user file.

comparison of which variables are most important in terms of predicting surgical treatment versus survival, a Cox Model was fitted using all the predictors used to create the SSS but not the SSS itself.

Distribution of the SSS and the Kaplan-Meier estimate of 3-year survival were visually illustrated for each stage by treatment category (surgical vs nonsurgical) and within each stage the mean values of SSS were statistically compared for the 2 treatment categories. To assess the potential benefit of surgery for patients with high SSS (above approximately the $75 \%$ percentile), the overall effect of surgery on the hazard of death was estimated for these patients by the Cox model separately by stage. These models were further adjusted for SSS by categorizing into small subgroups each representing $5 \%$ of the top quartile. $P$ values less than .05 were considered statistically significant. All statistical analyses were conducted using SAS, version 9.4 (SAS Institute Cary, NC). This study received a determination letter from the University of California, Davis Institutional Review Board.

\section{RESULTS}

We identified 300,572 patients with biopsy-proven stage IIIA, IIIB, or IV NSCLC from the NCDB (Figure 1). Notably, $18,701(6.2 \%)$ of these patients had surgery as part of their treatment regimen. In total, 265,183 patients had survival data available for follow-up for use in Cox proportional hazards model. The patients with missing survival information were evenly distributed across the surgery and nonsurgery groups and SSS. As previously reported, the strongest predictors of selection for surgery were AJCC clinical metastatic group, AJCC clinical nodal group, and age. ${ }^{8}$ The strongest predictors affecting the hazard of death were AJCC clinical metastatic group and age (Table 1).

In Cox proportional hazards model, SSS was highly predictive of OS (Table 2). The C-index for a model based on the entire cohort was 0.89 , and predictive ability increased when each stage group was considered separately. OS was also significantly greater in the surgically treated patients across all stage groups $(P<.001)$ (Figure 2$)$, with median survival times for surgical patients about 3-fold longer (Table 3). Within each stage, surgical patients show both greater values of SSS (within each stage, mean values for SSS are significantly different between the surgical and nonsurgical group with $P<.0001)$ and a greater probability of 3-year survival than their nonsurgical counterparts (Figure 3).

A substantial number of nonsurgical patients, however, have high SSS values, comparable with the surgical group. We assessed the potential impact of surgery on the hazard of death using the Cox model separately by stage, restricting analysis to approximately the upper quartile of SSS (900 for stage IIIA, 800 for stage IIIB, 700 for stage IV) and adjusting for narrowly defined SSS categories. Within this group, across all stages, the hazard rate was at least 2 times greater for patients not treated surgically compared with patients with similar SSS who received surgical treatment. The hazard ratios (HR) were stage IIIA: HR, 2.1; $95 \%$ CI, 2.0-2.2, stage IIIB: HR, 2.3; 95\% CI, 2.2-2.5, and stage IV: HR; 2.3; 95\% CI, 2.2-2.4.

\section{DISCUSSION}

Using clinical, demographic, and pathologic variables, we have created a novel prediction score that estimates the probability of surgical treatment and OS accurately in advanced-stage NSCLC. Across all stages, there is a meaningful survival advantage for surgical patients. The SSS uses the AJCC staging system variables supplemented by selected clinical variables that are available at the time of 
TABLE 1. Association between hazard of death and the patient variables used to create the Surgical Selection Score

\begin{tabular}{|c|c|}
\hline Variable & $\begin{array}{c}\text { Hazard ratio } \\
\text { (95\% confidence } \\
\text { interval) }\end{array}$ \\
\hline \multicolumn{2}{|l|}{ Clinical T status } \\
\hline 1 & $0.77(0.76-0.78)$ \\
\hline 2 & $0.83(0.82-0.84)$ \\
\hline 3 & $0.87(0.86-0.88)$ \\
\hline 4 & Reference \\
\hline $\mathrm{X}$ & $0.97(0.96-0.99)$ \\
\hline \multicolumn{2}{|l|}{ Tumor size } \\
\hline T1a & $0.72(0.70-0.73)$ \\
\hline $\mathrm{T} 1 \mathrm{~b}$ & $0.74(0.73-0.75)$ \\
\hline $\mathrm{T} 2 \mathrm{a}$ & $0.79(0.78-0.80)$ \\
\hline $\mathrm{T} 2 \mathrm{~b}$ & $0.87(0.86-0.89)$ \\
\hline $\mathrm{T} 3$ & Reference \\
\hline \multicolumn{2}{|l|}{ Clinical N status } \\
\hline 0 & $0.75(0.74-0.77)$ \\
\hline 1 & $0.84(0.82-0.85)$ \\
\hline 2 & $0.92(0.91-0.93)$ \\
\hline 3 & Reference \\
\hline $\mathrm{X}$ & $1.00(0.99-1.02)$ \\
\hline \multicolumn{2}{|l|}{ Clinical M status } \\
\hline 0 & $0.46(0.45-0.46)$ \\
\hline 1 & $0.92(0.91-0.94)$ \\
\hline $1 \mathrm{~A}$ & $0.68(0.66-0.70)$ \\
\hline 1B & Reference \\
\hline \multicolumn{2}{|l|}{ Histology } \\
\hline Squamous cell carcinoma & $0.91(0.90-0.92)$ \\
\hline Adenocarcinoma & $0.86(0.85-0.87)$ \\
\hline NSCLC, NOS & Reference \\
\hline \multicolumn{2}{|l|}{ Age group, y } \\
\hline$<52$ & $0.55(0.54-0.56)$ \\
\hline$\geq 52-59$ & $0.59(0.58-0.60)$ \\
\hline $59-67$ & $0.63(0.62-0.64)$ \\
\hline $67-75$ & $0.68(0.68-0.69)$ \\
\hline $75-\leq 81$ & $0.81(0.79-0.82)$ \\
\hline$>81$ & Reference \\
\hline \multicolumn{2}{|l|}{ Charlson-Deyo Index } \\
\hline 0 & Reference \\
\hline 1 & $1.20(1.19-1.21)$ \\
\hline 2 & $1.42(1.41-1.44)$ \\
\hline \multicolumn{2}{|l|}{ Race } \\
\hline White & $1.04(1.03-1.06)$ \\
\hline Other & $0.82(0.79-0.84)$ \\
\hline Black & Reference \\
\hline \multicolumn{2}{|l|}{ Insurance status } \\
\hline Private & $0.80(0.79-0.82)$ \\
\hline Medicare & $0.91(0.89-0.93)$ \\
\hline Medicaid & $0.96(0.93-0.98)$ \\
\hline Other government & $0.93(0.89-0.97)$ \\
\hline Not insured & Reference \\
\hline \multicolumn{2}{|l|}{ Income } \\
\hline High & $0.92(0.91-0.93)$ \\
\hline
\end{tabular}

TABLE 1. Continued

\begin{tabular}{|c|c|}
\hline Variable & $\begin{array}{c}\text { Hazard ratio } \\
\text { (95\% confidence } \\
\text { interval) }\end{array}$ \\
\hline Middle & $0.97(0.96-0.98)$ \\
\hline Low & Reference \\
\hline \multicolumn{2}{|l|}{ Facility type } \\
\hline Academic/research program & $0.87(0.86-0.88)$ \\
\hline $\begin{array}{l}\text { Comprehensive community } \\
\text { cancer program }\end{array}$ & $0.95(0.94-0.97)$ \\
\hline Community cancer program & Reference \\
\hline
\end{tabular}

NSCLC, Non-small cell lung cancer; NOS, not otherwise specified.

treatment decisions to estimate probability of surgical treatment and survival in a stage-specific manner. It has excellent discriminatory capabilities, as shown by $\mathrm{C}$-indices approaching 1 for both the outcomes described in our previous publication and in the current analysis. In the current analysis, we defined the stage-specific ability of the SSS to predict OS for patients with advanced-stage NSCLC and assessed the impact of surgery in patients with same stage and very comparable high SSS in an attempt to mitigate the influence of selection bias.

Our data support a therapeutic effect of surgery for at least some of these patients beyond what can be explained by selection bias, given the high precision of the SSS to predict surgical treatment and the improved OS in surgical patients at comparable high SSS. In our view, these findings warrant further investigation to understand the potential favorable effects of surgery in appropriately selected patients with advanced-stage NSCLC. As mentioned previously, there are data to suggest improved survival in patients with advanced-stage NSCLC who undergo pulmonary resection. A recent study using NCDB data that reflected treatments given for stage IIIA NSCLC from 1998 to 2010 demonstrated a low incidence of pneumonectomy $(16 \%)$ and superior survival outcomes for surgical patients who were matched to nonsurgical patient using demographic variables (median survival 35.9 for trimodality patients vs 19.7 months for chemotherapy and radiation patients, $P<.001) .{ }^{6}$ In a study of stage IIIB patients using data from the SEER database for 2004 to 2012, median OS was 29 months in patients treated with surgery and radiation compared with only 11 months in patients treated with radiation alone $(P<.0001){ }^{14}$

TABLE 2. C-index for the Surgical Selection Score for prediction of overall survival

\begin{tabular}{lcc}
\hline \multicolumn{1}{c}{ Stage } & C-index & $\mathbf{9 5} \%$ confidence interval \\
\hline IIIA, IIIB, IV & 0.89 & $0.891-0.896$ \\
IIIA & 0.91 & $0.908-0.919$ \\
IIIB & 0.94 & $0.938-0.947$ \\
IV & 0.94 & $0.941-0.946$ \\
\hline
\end{tabular}



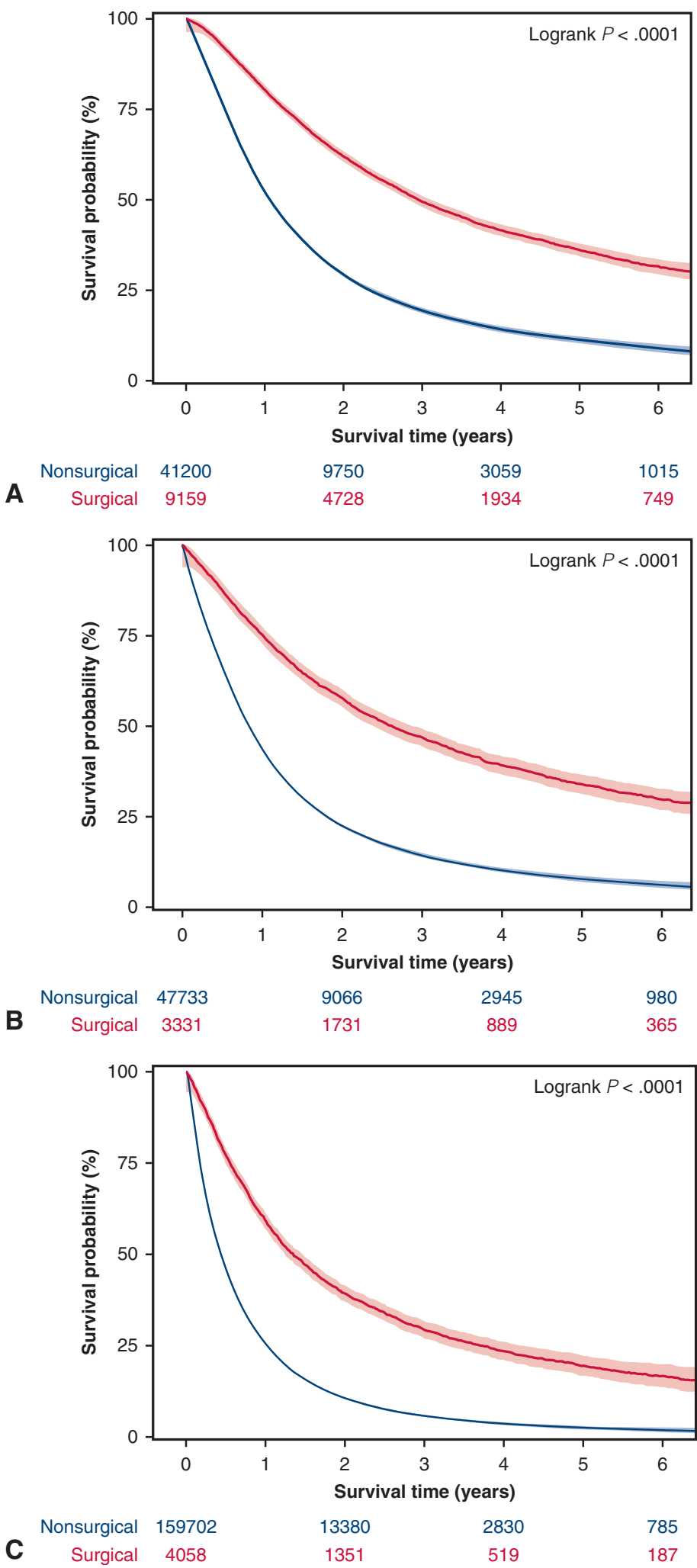

FIGURE 2. Kaplan-Meier analysis of patients stratified by stage. A, Stage IIIA; B, stage IIIB; and C, stage IV patients treated surgically have significantly longer overall survival across all stages. 
TABLE 3. MST by treatment group

\begin{tabular}{|c|c|c|}
\hline & \multicolumn{2}{|c|}{ MST $(95 \%$ confidence interval $)$, mo } \\
\hline & Surgical & Nonsurgical \\
\hline Stage IIIA & $35.5(34.3-36.9)$ & $12.9(12.7-13.0)$ \\
\hline Stage IIIB & $31.4(29.4-33.6)$ & $10.0(9.9-10.2)$ \\
\hline Stage IV & $16.2(15.2-17.3)$ & $5.3(5.3-5.4)$ \\
\hline
\end{tabular}

MST, Median survival time.

Similarly, Bateni and colleagues ${ }^{15}$ found that lung resection in patients with stage IV cancer is safe and associated with similar 30-day mortality and serious morbidity when compared with patients without disseminated malignancy in the American College of Surgeons National Surgical Quality Improvement Program dataset. Although these studies attempted to control for selection bias with statistical techniques, similar to ours, they are limited by their retrospective nature and inability to assess for confounding variables that are not captured in these large datasets. ${ }^{7}$

Despite these limitations, there are significant differences in the OS trends in these series compared with the earlier randomized trials, reinforcing the concept that improvements in staging and multimodality therapies, including surgical outcomes, may identify a small subset of advanced-staged patients who may benefit from surgical management. In concert with these data, the SSS is applicable to each of stage IIIA, IIIB, and IV NSCLC patients and continues to highlight the potential benefit of including surgery in the treatment of advanced-stage patients, including stage IIIB patients.

Importantly, although current National Comprehensive Cancer Network guidelines endorse limited recommendations for surgical treatment for patients with advancedstage NSCLC, these guidelines are drawn from studies that have not adequately evaluated the heterogeneity among patients with advanced-stage NSCLC. Moreover, these studies do not reflect contemporary surgical outcomes using current minimally invasive surgical techniques and strategies to minimize the use of highly morbid pneumonectomy. ${ }^{4,16,17}$ For example, in a randomized trial of induction chemotherapy followed by surgery or radiotherapy for patients with stage IIIA-N2 NSCLC, representing treatments given from 1994 to 2002, van Meerbeeck and colleagues ${ }^{17}$ reported exploratory thoracotomy in $14 \%$, pneumonectomy in $47 \%$, and incomplete resection in 50\% of patients. Therefore, it is not surprising that the authors observed no differences in OS between the surgical and radiotherapy cohorts. ${ }^{17}$ Similarly, Albain and colleagues ${ }^{16}$ compared induction chemotherapy plus radiation followed by surgery to induction chemotherapy plus definitive radiotherapy in a randomized trial of stage IIIA-N2 patients. These authors also found no significant difference in OS between the surgical and nonsurgical groups. However, this trial also was noted to have a high rate of pneumonectomy
$(34 \%)$, and $9 \%$ of patients underwent no or incomplete resection. In addition, their patient sample was heterogeneous, with $20 \%$ having 2 positive nodal stations and $2 \%$ having 3 positive nodal stations. Consequently, it is likely that patient heterogeneity and poor surgical outcomes did not allow for a fair comparison between surgically and nonsurgically treated groups as well as underestimate survival outcomes observed with surgery in a more contemporaneous cohort such as ours. These data should raise questions about the role of thoracic surgery for carefully selected patients with advanced-stage NSCLC in the future and suggest the need for randomized trials applying contemporary surgical techniques.

Because of the heterogeneous nature of patients with advanced-stage NSCLC and multiplicity of treatment options, clinical trials to assess the true therapeutic impact of surgery for these patients are likely prohibitively challenging to design and accrue. Yet, delivery of an effective treatment modality, including surgery, to an appropriately selected patient subset who will derive benefit is the cornerstone of personalized medicine. Unlike retrospective database analyses, which only allow for prediction of outcomes after treatment decisions have been made, we maintain that a significant strength of the SSS is its ability to be used prospectively to assess a patient's probability of both receiving surgical treatment and deriving improved OS. ${ }^{18-20}$

Moreover, given its novel ability to be used prospectively, after further validation, the SSS may have a role in reducing treatment variation because it can be used by both surgeons and nonsurgeons to identify patients who may benefit from consideration for surgical treatment and/or enrollment into prospective clinical trials stratified by SSS. We do not contend that the SSS should be used as a dichotomous tool to decide who can and cannot undergo surgery as part of multimodality treatment. Rather, we believe it may help nonsurgeons identify additional patients who could be evaluated for surgery and that surgeons would provide the appropriate evaluation of surgical candidacy to include a physiologic assessment, tumor resectability, and assessment of disease burden, as per guidelines. ${ }^{4}$ Multidisciplinary tumor boards and telehealth interventions could then be used to evaluate and extend surgical options to patients who might not otherwise be exposed. ${ }^{21,22}$

Despite its strengths, it is important to acknowledge the limitations of our analysis. The SSS is limited by the ongoing presence of unmeasured confounding variables. Because tumor resectability, pulmonary function, and smoking status are not captured in administrative datasets such as the NCDB, the SSS cannot definitively predict which patients will be appropriate for surgical resection. These factors may have biased our results in favor of surgery despite controlling for comorbidities using the 

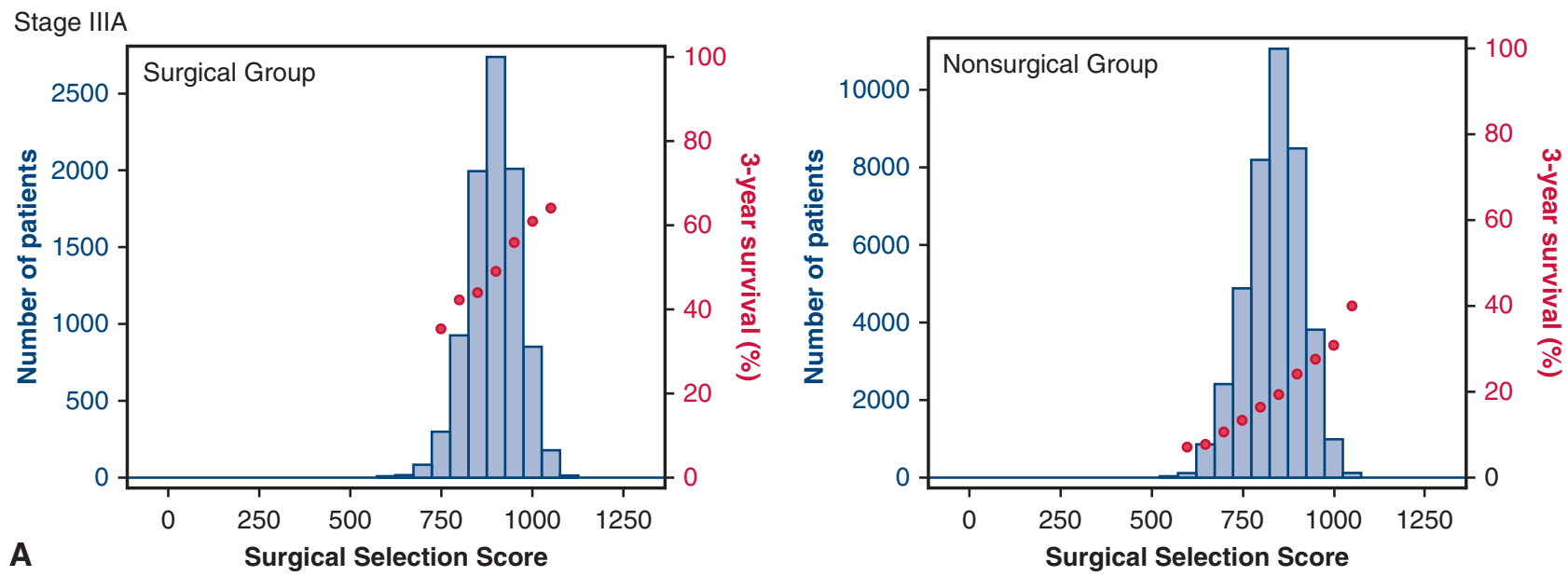

Stage IIIB
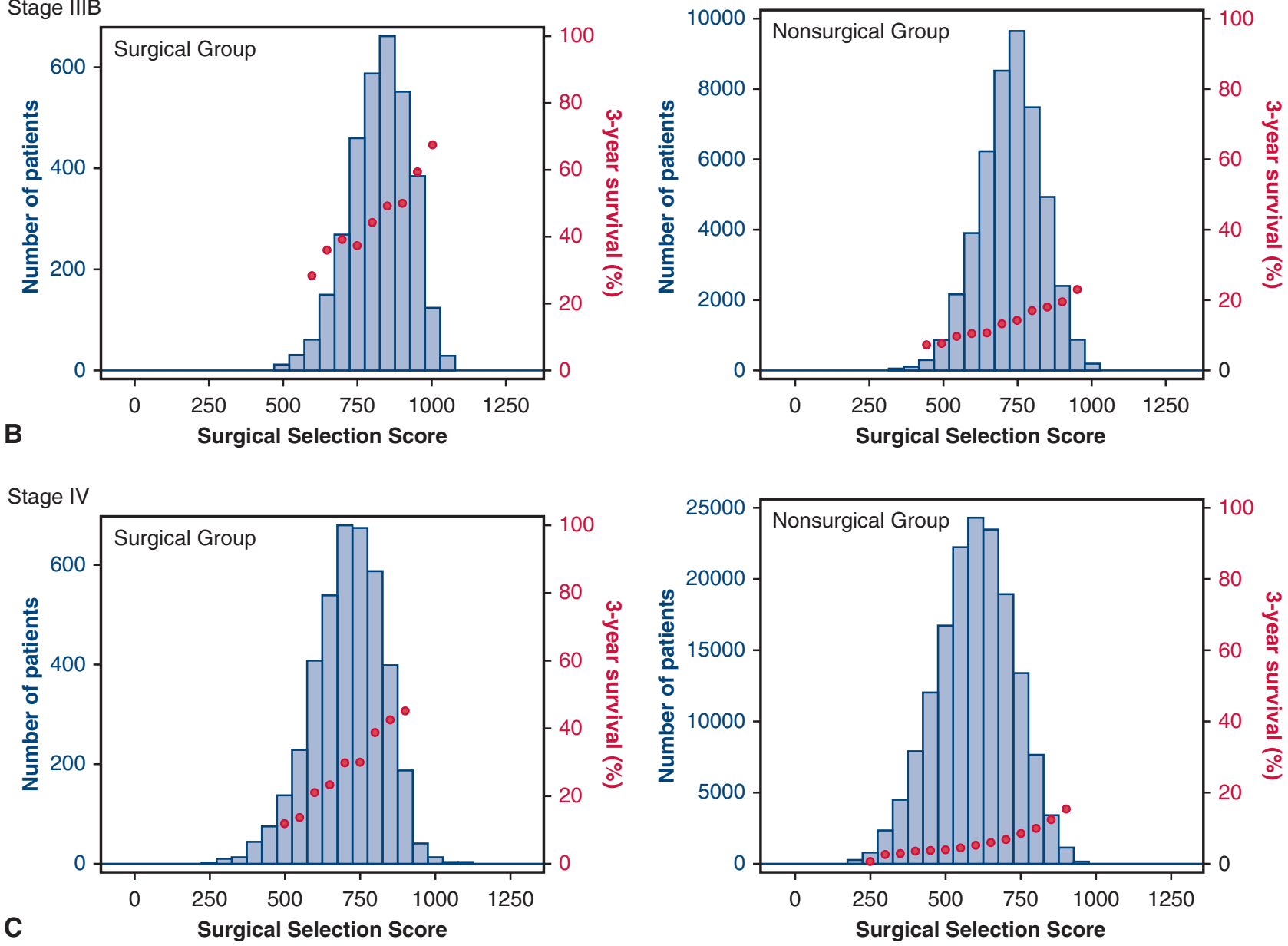

FIGURE 3. Distribution of Surgical Selection Score and Kaplan-Meier 3-year survival probability. A, Stage IIIA; B, stage IIIB; C, and stage IV patients have significantly longer survival when surgery is included in their treatment regimens.

Charlson index, which is the only available measure of performance status in the NCDB. In addition, disease burden and intent of resection are not currently captured in the NCDB and would impact the decision to offer therapeutic surgical resection to a patient. It is also likely that response to systemic treatment and molecular status of tumors were considered in surgical decisions, but there is no large dataset that currently capture these data or allow it to be analyzed. Therefore, future analyses incorporating these data will be important to validate our 
findings and thereby optimize personalized treatment for these patients.

In a manuscript that is heavily dependent on statistical analysis, we must acknowledge statistical limitations. Patients missing essential characteristics like the Charlson index or survival information (when needed) were omitted from the cohort before the statistical analysis, and we did not attempt to impute missing values because we believe it is reasonable to assume these data were missing at random. It is a limitation that we had to omit incomplete data and we cannot be certain that data was missing at random even though patients with missing survival information were evenly distributed across the surgery and nonsurgery groups and also SSS strata.

Although it is possible that there are patients with high SSS who are not candidates for surgical resection, it is also possible that the SSS will identify patients who will derive benefit from surgery but were previously not offered this modality. We acknowledge that our analysis using the SSS cannot fully eliminate the impact of selection bias on the survival outcomes observed, but the magnitude of the clinically and statistically significant survival differences between the groups, particularly when controlling for SSS, suggest that further exploration of the association of surgery with improved survival is warranted. In the era of increasingly effective systemic therapies, the incorporation of surgery into a careful multimodality treatment approach may improve disease control and OS beyond what can be attributed to selection bias alone.

\section{CONCLUSIONS}

The management of advanced-stage NSCLC is undergoing a paradigm shift, and patients whose prognosis was previously measured in months can now often be measured in years. We have derived an SSS that identifies factors that robustly predict selection for surgical management and OS in patients with stage IIIA, IIIB, and IV NSCLC. Therefore, our data suggest that the SSS may prove to be a valuable tool for clinicians to help identify patients who could potentially benefit from surgical evaluation in an era in which treatment options for advanced-stage NSCLC are expanding, but overall treatment nihilism appears to persist for lung cancer patients across all stages. ${ }^{2,23-25}$

\section{Conflict of Interest Statement}

Authors have nothing to disclose with regard to commercial support.

We thank Dr Richard Bold for his assistance and support of this project. The National Cancer Database (NCDB) is a joint project of the Commission on Cancer $(\mathrm{CoC})$ of the American College of Surgeons and the American Cancer Society. The CoC's NCDB and the hospitals participating in the $\mathrm{CoC} \mathrm{NCDB}$ are the source of the deidentified data used herein; they have not verified and are not responsible for the statistical validity of the data analysis or the conclusions derived by the authors.

\section{References}

1. SEER Stat Fact Sheets: Lung and Bronchus Cancer. Surveillance, Epidemiology and End Results program cancer statistics. Available at: http://seer.cancer.gov/ statfacts/html/lungb.html. Accessed February 28, 2018.

2. David EA, Daly ME, Li C-S, Chiu CL, Cooke DT, Brown LM, et al. Increasing rates of no treatment in advanced stage NSCLC patients: a propensity matched analysis. J Thorac Oncol. 2017;12:437-45.

3. David EA, Canter RJ, Chen Y, Cooke DT, Cress RD. Surgical management of advanced stage NSCLC is decreasing but remains associated with improved survival. Ann Thorac Surg. 2016;102:1101-9.

4. NCCN guidelines for NSCLC. Available at: http://www.nccn.org/professionals/ physician_gls/pdf/nscl.pdf. Accessed January 1, 2018.

5. Bott MJ, Patel AP, Crabtree TD, Morgensztern D, Robinson CG, Colditz GA, et al. Role for surgical resection in the multidisciplinary treatment of stage IIIB non-small cell lung cancer. Ann Thorac Surg. 2015;99:1921-8.

6. Patel AP, Crabtree TD, Bell JM, Guthrie TJ, Robinson CG, Morgensztern D, et al. National patterns of care and outcomes after combined modality therapy for stage IIIA non-small-cell lung cancer. $J$ Thorac Oncol. 2014;9:612-21.

7. Boffa DJ, Rosen JE, Mallin K, Loomis A, Gay G, Palis B, et al. Using the National Cancer Database for outcomes research: a review. JAMA Oncol. 2017;3: 1722-8.

8. David EA, Andersen SW, Beckett LA, Melnikow J, Kelly K, Cooke DT, et al. A model to predict the use of surgical resection for advanced-stage non-small cell lung cancer patients. Ann Thorac Surg. 2017;104:1665-72.

9. Bilimoria K, Stewart A, Winchester D, Ko C. The National Cancer Data Base: a powerful initiative to improve cancer care in the United States. Ann Surg Oncol. 2008;15:683-90

10. Deyo RA, Cherkin DC, Ciol MA. Adapting a clinical comorbidity index for use with ICD-9-CM administrative databases. J Clin Epidemiol. 1992;45:613-9.

11. Penciana MJ, D'Agostino RB. Overall $\mathrm{C}$ as a measure of discrimination in survival analysis: model specific population value and confidence interval estimation. Stat Med. 2004;23:2109-23.

12. Pencina MJ, D'Agostino RB Sr. Evaluating discrimination of risk prediction models: the c statistic. JAMA. 2015;314:1063-4.

13. Harrel F, Lee K, Mark D. Multivariable prognostic models: issues in developing models, evaluating assumptions and adequacy, and measuring and reducing errors. Stat Med. 1996;15:361-87.

14. Herskovic A, Chitti B, Christos P, Wernicke AG, Parashar B. Addition of surgery after radiation significantly improves survival in stage IIIB non-small cell lung cancer: a population-based analysis. World J Surg. 2016;41:758-62.

15. Bateni SB, David EA, Bold RJ, Cooke DT, Meyers FJ, Canter RJ. Lung resection is safe and feasible among stage IV cancer patients: an American College of Surgeons National Surgical Quality Improvement Program analysis. Surgery. 2017; 161:1307-14.

16. Albain KS, Swann RS, Rusch VW, Turrisi AT III, Shepherd FA, Smith C, et al Radiotherapy plus chemotherapy with or without surgical resection for stage II non-small-cell lung cancer: a phase III randomised controlled trial. Lancet. 2009; 374:379-86.

17. van Meerbeeck JP, Kramer GWPM, Van Schil PEY, Legrand C, Smit EF, Schramel F, et al. Randomized controlled trial of resection versus radiotherapy after induction chemotherapy in stage IIIA-N2 non-small-cell lung cancer. $J$ Natl Cancer Inst. 2007;99:442-50.

18. Young KA, Efiong E, Dove JT, Blansfield JA, Hunsinger MA, Wild JL, et al. External validation of a survival nomogram for non-small cell lung cancer Using the National Cancer Database. Ann Surg Oncol. 2017; 24:1459-64.

19. Hui Z, Dai H, Liang J, Lv J, Zhou Z, Feng Q, et al. Selection of proper candidates with resected pathological stage IIIA-N2 non-small cell lung cancer for postoperative radiotherapy. Thorac Cancer. 2015;6:346-53.

20. Liang W, Zhang L, Jiang G, Wang Q, Liu L, Liu D, et al. Development and validation of a nomogram for predicting survival in patients with resected non-smallcell lung cancer. J Clin Oncol. 2015;33:861-9.

21. Clark JM, Heifetz LJ, Palmer D, Brown LM, Cooke DT, David EA. Telehealth allows for clinical trial participation and multimodality therapy in a rural patient with stage 4 non-small cell lung cancer. Cancer Treat Res Commun 2016;9:139-42. 
22. Kehl KL, Landrum MB, Kahn KL, Gray SW, Chen AB, Keating NL. Tumor board participation among physicians caring for patients with lung or colorectal cancer. J Oncol Pract. 2015;11:e267-78.

23. Ellis PM. The importance of multidisciplinary team management of patients with non-small-cell lung cancer. Curr Oncol. 2012;19(suppl 1):S7-15.

24. Vest MT, Herrin J, Soulos PR, Decker RH, Tanoue L, Michaud G, et al. Use of new treatment modalities for non-small cell lung cancer care in the medicare population. Chest. 2013;143:429-35.
25. Dawe DE, Pond GR, Ellis PM. Assessment of referral and chemotherapy treatment patterns for elderly patients with non-small-cell lung cancer. Clin Lung Cancer. 2016;17:563-72.e2.

Key Words: NSCLC, advanced stage, surgery, multimodality treatment, survival, chemotherapy, radiation

Readers who found these articles interesting may also like to read the following papers found in recent and future issues of our sister publications, Seminars in Thoracic and Cardiovascular Surgery and Operative Techniques in Thoracic and Cardiovascular Surgery!

\section{Thoracic: Lung Cancer}

ORIGINAL SUBMISSION: Trends and Outcomes of Thoracoscopic Lobectomy or Segmentectomy: A National Surgical Quality Improvement Project Analysis. Ikenna Okereke. Semin Thoracic Surg 2018: in Press.

Editorial Commentary: \#vatslobectomy: Daniel Miller: Semin Thoracic Surg 2018: in Press.

ORIGINAL SUBMISSION: Microcoil-Guided Video Assisted Thoracoscopic Excision of Nodules suspicious for Metastasis in Patients with Extra-Thoracic Malignancies. Omamah Almousa. Semin Thoracic Surg 2018: in Press.

Editorial Commentary: Will the Real Slim Shady Please Stand Up? DuyKhanh P. Ceppa. Semin Thoracic Surg 2018: in Press. ORIGINAL SUBMISSION: Three-dimensional Navigation for Thoracoscopic Sublobar Resection Using a Novel Wireless Marking System. Yojiro Yutaka. Semin Thoracic Surg 2018: 230-237.

Editorial Commentary: Searching for the Holy Grail -and Small Pulmonary Nodules. Chadrick E. Denlinger. Semin Thoracic Surg 2018: 238-239.

ORIGINAL SUBMISSION: Broncho-Pleural Fistula after Pneumonectomy: Risk Factors and Management, Focusing on Open Window Thoracostomy. Antonio Mazzella. Semin Thoracic Surg 2018: 104-113.

Editorial Commentary: Management of Post-Pneumonectomy Bronchopleural Fistula: a Roadmap for Rescue. Virginia R. Litle. Semin Thoracic Surg 2018: 114-115.

ORIGINAL SUBMISSION: Intraoperative Computed Tomography Navigation During Thoracoscopic Segmentectomy for Smallsized Lung Tumors. Sung Soo Chang. Semin Thoracic Surg 2018: 96-101.

Editorial Commentary: Much Ado About Precision. Michael Ebright. Semin Thoracic Surg 2018: 102-103.

ORIGINAL SUBMISSION: A New Instrument for Intraoperative Visualization of Safe Surgical Margins for Small Lung Nodules Gaetano Rocco. Semin Thoracic Surg 2018: 92-94.

Editorial Commentary: Sublobar Resections -the Margins Justify the Means. Stephen R. Broderick. Semin Thoracic Surg 2018: 95 ORIGINAL SUBMISSION: Robotic Right Middle Lobectomy. Bernard J. Park. Oper Tech Thorac Cardiovasc 2017: 2-16.

ORIGINAL SUBMISSION: Robotic Right Lower Lobectomy. Mark R. Dylewski. Oper Tech Thorac Cardiovasc 2017: 17-42.

ORIGINAL SUBMISSION: Robotic Lobectomy: Left Lower Lobectomy by Surgery. Carmelina Cristina Zirafa. Oper Tech Thorac Cardiovasc 2017: 43-57. 